\title{
sige \\ La fecha de la cerámica más temprana en los Andes sur. Una perspectiva macrorregional mediante modelos bayesianos
} RMA

The date of the earliest pottery in the Southern Andes: a macroregional perspective based on bayesian models

\author{
VI Jornadas \\ Arqueológicas Cuyanas
}

Erik J. Marsh

CONICET. Laboratorio de Paleo-Ecología Humana, Universidad Nacional de Cuyo, Mendoza, Argentina. Email: emarsh@mendoza-conicet.gob.ar

\begin{abstract}
Resumen
La cerámica más temprana marca un hito en la historia humana de los Andes del sur. Este trabajo propone una aproximación de la edad de la cerámica más temprana mediante modelos cronológicos basados en la estadística bayesiana. En estos, se comparan 84 fechados radiocarbónicos y de termoluminiscencia de 49 sitios que abarcan dos áreas en Argentina dentro de las provincias de San Juan y Mendoza y tres áreas del norte semi-árido, centro y centro-sur de Chile. Los datos analizados indican un patrón llamativo de fechas similares en cuatro de las cinco áreas, con medianas entre 2100 y 2180 cal. AP (2250-1920, 68\% probabilidad, límites iniciales), aunque algunos de los rangos de error son amplios. Se destaca la similitud de las fechas en una macrorregión que se extiende a lo largo de 1000 kilómetros (norte-sur) entre las $30^{\circ}$ a $39^{\circ} \mathrm{S}$ y que contiene una gran variedad de ambientes. La coincidencia temporal resulta aún más llamativa porque el límite sur de agricultura en el continente Sudamericano se encuentra en estas áreas. Este trabajo pretende incentivar el interés de los investigadores en la excavación y el fechado de contextos relevantes y estimular las discusiones sobre la naturaleza de la adopción inicial de la cerámica en los Andes del sur.
\end{abstract}

Palabras clave: cerámica inicial; modelos bayesianos; fechados radiométricos; Andes del sur.

\begin{abstract}
The earliest pottery marks a milestone in the human history of the southern Andes. This paper proposes an approximation of the date of the earliest pottery using Bayesian chronological models. Models of 84 radiocarbon and thermoluminescence dates from 49 sites are compared among five areas in Argentina (the provinces of San Juan and Mendoza) and Chile (the semi-arid north, central, and south central). Although there are still few data, they show an intriguing pattern of very similar dates with medians between 2100-2180 cal BP (2250-1920,68\% probability, starting boundaries) in four of the areas, though some error ranges are large. The similarity of the dates is striking considering that the macro-region extends over one thousand kilometers (north-south) from $30^{\circ}$ to $39^{\circ} \mathrm{S}$ and includes a diverse range of environments. The contemporaneity is even more notable because these areas include the continent's southern limit of agriculture. It is hoped this will encourage researchers to more carefully assess this period when excavating and dating relevant contexts as well as encourage discussions on the nature of the initial adoption of ceramics in the southern Andes.
\end{abstract}

Keywords: earliest ceramics; Bayesian models; radiometric dates; southern Andes.

Los orígenes de la cerámica se encuentran entre los temas clásicos de la arqueología (Barnett y Hoopes 1995; Rice 1999). Una visión ampliamente difundida surgió con el modelo popularizado por Childe (1925) de la revolución neolítica. Se propuso que la cerámica fue desarrollada por primera vez para utilizarse junto con la agricultura como parte de una vida sedentaria. Estos elementos del 'paquete' neolítico parecen haber ocurrido en forma simultánea debido al enfoque europeo, los modelos teleológicos de la evolución social y las cronologías poco precisas, muchas veces divididas en bloques temporales de más de 1.000 años. Esta propuesta neolítica tiene una inercia histórica en la arqueología mundial que continúa hasta el presente (p. ej., Jordan y Zvelebil 2009a; Pluciennik 2002, 2005; Rudebeck 2000). Sin embargo, las investigaciones actuales de diferentes partes del

Recibido 19-02-2016. Recibido con correcciones 11-01-2017. Aceptado 11-01-2017

Revista del Museo de Antropología, Suplemento Especial 1: 83-94, 2017 / ISSN 1852-060X (impreso) / ISSN 1852-4826 (electrónico) 
mundo muestran que los primeros recipientes cerámicos fueron inventados, fabricados y usados por cazadoresrecolectores estacionalmente sedentarios. Muchas de esas ocupaciones 'redundantes' (Simms et al. 1997) ocurrieron en nichos ambientales del viejo mundo que ofrecen la materia prima para confeccionar la cerámica junto con recursos alimenticios aptos (Jordan y Zvelebil 2009b). En varios casos, la cerámica se mantuvo como un componente estable de la vida nómada durante miles de años sin que se desarrollara la agricultura ni ocupaciones sedentarias, como en el emblemático caso de Japón (Kaner 2009), muchas regiones de Asia (capítulos en Jordan y Zvelebil 2009b), América del Norte (Eerkens 2003, 2004, 2008) y en sociedades actuales también (Arnold 1985).

En América del Sur, la arqueología también fue condicionada por las expectativas neolíticas y su equivalente local, el denominado período Formativo (Lumbreras 2006; Hocsman et al. 2012; Olivera et al. 2012), a pesar de que muchos datos certifican que la aparición de los primeros cultivos, la cerámica más antigua y la agricultura de escala mayor no fueron coetáneos. Estas actividades se desarrollaron en diferentes periodos separados muchas veces por más de 1.000 años (Lavallee, 2006). Esto se refleja bien en los datos del noroeste argentino, donde las investigaciones recientes están haciendo un seguimiento independiente de la cerámica temprana (García 1995; García e Higa 2014), el grado de sedentarismo (Marshall 2006; Olivera 2001), prácticas de agricultura (Lema 2010, 2014; Yacobaccio y Korstanje 2007) y horticultura a través de macrorrestos botánicos e instrumentos de molienda (Babot 2006, 2011; Lema 2010; Núñez et al. 2009). Dado este panorama, una meta clave en los Andes es desglosar los elementos materiales asociados a los conceptos de 'Neolítico' y 'Formativo' (Lavallée 2006; Núñez y Santoro 2011; Hocsman et al. 2012; Olivera 2001; Olivera et al. 2012; López Campeny y Romano 2012).

Aunque la cerámica más antigua no es indicativa de otros cambios 'neolíticos', su aparición señala un cambio importante en los modos de vida de los cazadoresrecolectores, quienes no usaban, y aparentemente no tuvieron la necesidad, ni el interés, de fabricar recipientes cerámicos durante milenios. Sin embargo, en muchos casos, una vez que comenzaron con el uso de esta tecnología no dejaron de utilizarla. Por ello, la adopción de la cerámica es un hito en la historia de la cultura material de muchos grupos. La presencia de cerámica es un cambio material fundamental en el registro arqueológico resultando un punto esencial para evaluar otros posibles cambios asociados, como por ejemplo aumentos en la demografía y la complejidad social. La simple presencia de cerámica en el registro no es muy cuestionable y su análisis o estudio no varía entre las diversas corrientes teóricas o formaciones de los arqueólogos, lo cual representa una ventaja, permitiendo compilar y comparar estudios recientes con otros más antiguos.

En este marco, el presente trabajo apunta a enriquecer discusiones internacionales sobre: 1) la adopción de la cerámica a largo plazo por parte de cazadores-recolectores versus su adopción como parte de un 'paquete' neolítico (o en el caso de los Andes, el 'paquete' agropastoril) y 2) la difusión de la tecnología versus su invento independiente en regiones vecinas. Esas discusiones dependerán en gran medida de cronologías refinadas, en las que se basa el enfoque de los interrogantes principales del presente estudio: ¿cuándo se usó la cerámica por primera vez en cada una de las cinco áreas de los Andes del sur? ¿Se correlacionan las fechas entre sí? En este contexto se espera abrir una discusión sobre el lapso cronológico relacionado a la adopción de la cerámica y también aumentar el interés de los investigadores en la excavación y el fechado de contextos relacionados así como también en los debates sobre la naturaleza del proceso del cambio.

\section{Objetivo y antecedentes de regiones vecinas}

El presente trabajo pretende discutir la edad de aparición de la tecnología cerámica en una escala amplia que abarca los Andes del sur, con el objetivo de aportar al esclarecimiento de las causas y contextos de la incorporación de esta tecnología. Para esto se utiliza la información publicada de todos los sitios arqueológicos con cerámica temprana dentro de la región, los cuales son discutidos mediante el uso de modelos de estadística bayesiana. Los distintos trabajos en el sur del área Andina han mostrado cierta contemporaneidad en la aparición de la cerámica, tema que ha sido escasamente trabajado y necesita ser discutido en profundidad. Específicamente, el área de estudio abarca el sector cordillerano del oeste de las provincias de San Juan y Mendoza, Argentina y tres áreas del lado chileno: el norte semi-árido, central y centro-sur, siguiendo las divisiones tradicionales entre investigadores regionales. El área de estudio tiene 49 sitios y una extensión de 1000 kilómetros en sentido norte-sur, entre $30^{\circ}$ y $39^{\circ} \mathrm{S}$ de latitud (Figura 1).

Comparando los Andes del sur con otras regiones vecinas, la cerámica más antigua fue mucho más anterior en las regiones del norte y del este. Al sur, en Patagonia, su primer uso fue posterior y por lo general más marginal. Al norte, en los Andes centrales, la cerámica más antigua encontrada proviene de varios sitios de forma contemporánea dentro de la cuenca del Lago Titicaca, para la cual pudo estimarse a través del uso de modelos bayesianos, una mediana de 3440 cal. AP (3470-3400 cal. AP, 68\% probabilidad, calibrada con IntCal13 [Reimer et al. 2013]) (Marsh 2015:21). La cerámica más antigua es contemporánea con un aumento demográfico, la integración de prácticas agrícolas y pastoriles y la fundación de pueblos como parte de una vida mucho más sedentaria (Bandy 2001:280; Browman 1981; Hastorf 2008; Marsh 2016). En cambio, los grupos móviles 


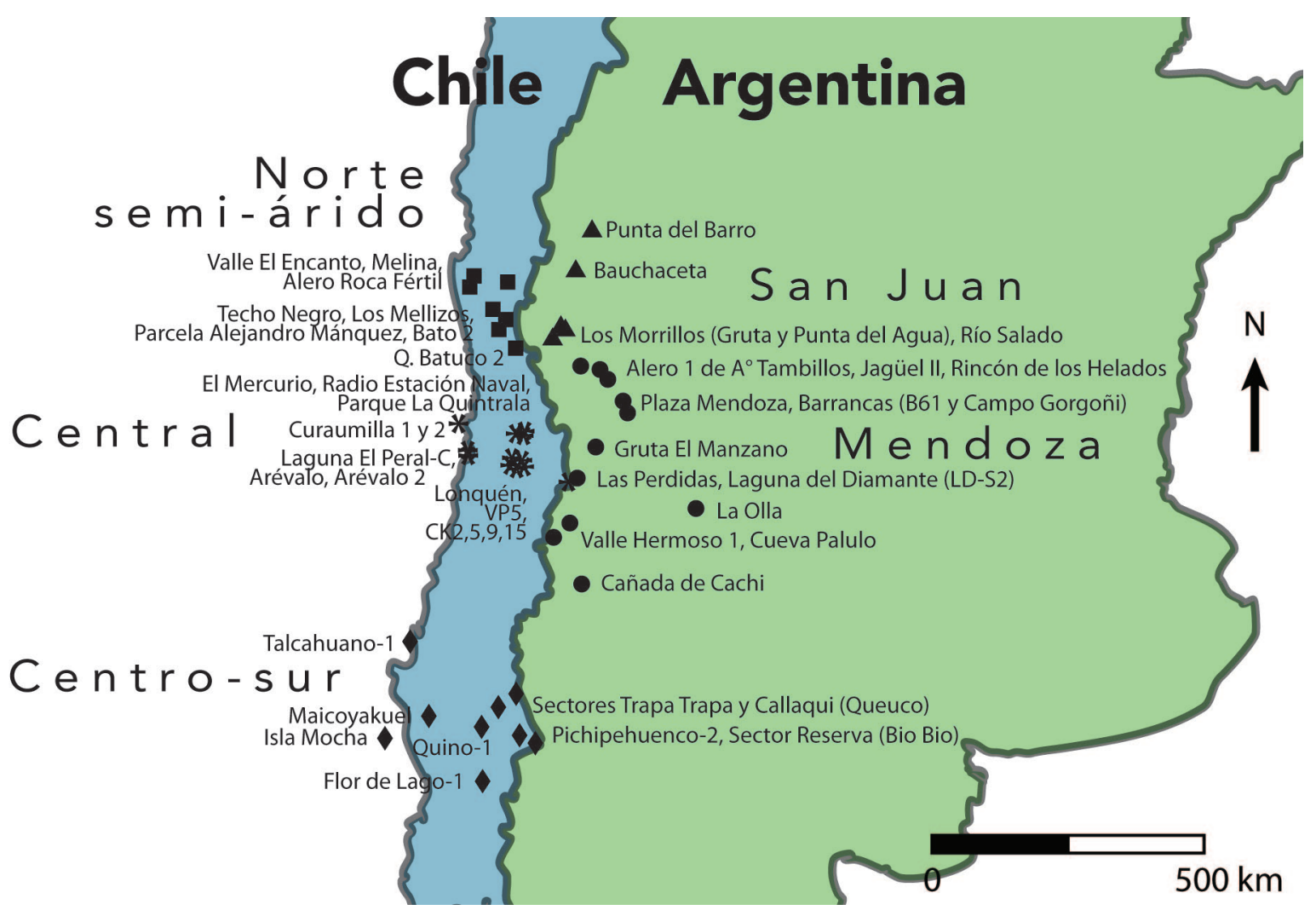

Figura 1. Mapa del cono sur de Sudamérica, con Argentina en verde y Chile en azul. Los sitios de San Juan se indican con triángulos, los de Mendoza con círculos, los del norte semi-árido chileno con cuadrados, los de Chile central con asteriscos y los del centro-sur chileno con diamantes.

Figure 1. Map of the southern cone of South America, with Argentina in green and Chile in blue. The sites from San Juan are marked as triangles, sites from Mendoza as circles, sites from the semi-arid region of Chile as squares, sites from central Chile as asterisks, and sites from south-central Chile as diamonds.

que habitaron el norte de Chile y el noroeste argentino durante estos siglos mantenían mayor movilidad al momento de la incorporación de la cerámica y los cultivos domésticos (Babot 2006; Lema 2010, 2014; Núñez et al. 2009). Los fechados más tempranos del norte de Chile y el noroeste argentino son de una edad similar o unos siglos posteriores a los hallados en la cuenca del Lago Titicaca (Fernández 1989; García 1995, García y Higa 2014; Hocsman 2002; Núñez y Santoro 2011; Olivera et al. 2015; Korstanje et al. 2015; Greco 2012). Al este, en las tierras bajas de la gran región pampeana, existen fechados similares para cerámicas, donde los grupos usaron la cerámica por primera vez 3340-3170 cal. AP (68\% probabilidad, CAMS-48494) en el sitio Zanjón Seco 2, una fecha que concuerda con otras de la región (Politis et al. 2001:173-176). Finalmente, al sur, los cazadores recolectores de Norpatagonia incorporaron la cerámica varios siglos después que en los Andes del sur (Sanguinetti de Bórmida et al. 2000; Schuster 2014; Silveira et al. 2013).

\section{Métodos y materiales: modelos bayesianos y fechados}

Los modelos de Bayes se utilizan para evaluar las tendencias cronológicas representadas por las distribuciones de probabilidad de los fechados. Este método ha sido usado por arqueólogos desde la década de los 90 (p. ej., Bayliss 2009; Bronk Ramsey 2008; Buck et al. 1996; Buck y Millard 2004; Wood 2015), quienes han demostrado su utilidad para refinar cronologías arqueológicas con la posibilidad de agregar otras clases de información, como por ejemplo las secuencias estratigráficas o similitudes estilísticas. En los últimos años, los modelos bayesianos han sido usados cada vez más (ver el número especial de World Archaeology, volumen 47, número 4, 2015) y también en todos los países andinos (Görsdorf y Reindel 2002; Greco 2012; Koons y Alex 2014; Korpisaari et al. 2014; Marsh 2012, 2015; Marsh y Cortegoso 2014; Michczyński y Pazdur 2003; Michczyński et al. 2003, 2007; Ogburn 2012; Unkel et al. 2007; Ziedler et al. 1998).

A diferencia de la matemática y la estadística tradicional, la estadística bayesiana permite trabajar con distribuciones de probabilidad (ver introducciones al método en Bayliss 2009; Bayliss et al. 2007). Una de sus varias ventajas es la posibilidad de estimar la fecha de eventos que no se pueden fechar directamente, como por ejemplo el inicio de una fase cronológica, que es el propósito 
principal del presente trabajo. En esta ocasión, se utiliza el programa OxCal 4.2 (Bronk Ramsey 2009) y la curva de calibración del hemisferio sur SHCal13 (Hogg et al. 2013). Se presentan las fechas en años calibrados antes del presente (AP), es decir, antes del año 1950 DC. Las probabilidades posteriores, o modeladas, se denotan con letra cursiva (Bayliss et al. 2007:5), indicando su rango de probabilidad y el evento modelado. En el texto se presentan los resultados de los límites iniciales de la fase cerámica, que puede ser el límite inicial del modelo o un límite ubicado entre las fases cerámicas y no cerámicas. Se redondean a la década más próxima. La Tabla 1 incluye los datos completos de los resultados. En todos los casos, se podrá mejorar la cantidad y la calidad de la información, pero lo más probable es que los datos nuevos refinen la fecha inicial dentro de los rangos de error estimados. Una ventaja fundamental del método es que la fecha límite de una fase no depende de los fechados cercanos al límite ni de un solo fechado temprano, sino del acuerdo estadístico de todos los datos analizados.

En el área de estudio de los Andes del sur, se utilizaron 84 fechados radiocarbónicos y de termoluminiscencia. La Tabla 1 resume los datos para cada área; el detalle de todos los fechados se presenta en el Suplemento. Los modelos procesados en OxCal están disponibles a solicitud de quien lo desee. Se incluyeron la totalidad de los sitios arqueológicos con fechados asociados a tiestos cerámicos previos a los 1700 años AP. En el caso del norte semi-árido chileno, el número de fechados fue bajo, entonces se incluyeron fechados previos a los 1600 años AP. En tres áreas (San Juan, el norte semi-árido chileno y Chile central), se usaron pocos fechados de contextos no cerámicos para poder definir mejor la fecha límite de la primera cerámica. En cuanto al criterio para usar o no un fechado, se revisó la información publicada para cada fechado con el fin de evaluar el grado de asociación entre la presencia de tiestos cerámicos y fechados. En base a la información disponible, se decidió incluir cada fechado en la fase cerámica o la anterior, que se compone de contextos sin cerámica. Esto es un desafío porque en muchos casos los niveles con la cerámica más antigua no tienen muchos tiestos. En estos niveles hubo que revisar la posibilidad, a veces indicada por los respectivos autores, de que los fragmentos provengan de niveles superiores y no se asocien con el fechado del nivel.

\section{Resultados: fechados y modelos bayesianos de las cinco áreas}

\section{San Juan}

Para la provincia de San Juan, se generó un modelo compuesto basado en tres secuencias estratigráficas de las grutas 2 y 3 del sitio Los Morillos y la gruta 2 del Río Salado (Gambier 1977:27-31, 54-55). En cada una de las secuencias, hay fechados de los niveles inmediatamente por encima y por debajo del nivel que contiene la cerámica más temprana, cuya fecha es interpolada por el modelo. Además de esas tres fechas interpoladas, se agregan seis fechas más de la cerámica inicial proveniente de los sitios de Punta del Agua, Punta del Barro y Bauchaceta. Es llamativo que, en todos los contextos considerados, las fechas se superponen sin modelación alguna. Se excluye el fechado más temprano de Punta del Barro (GaK-8832), dado que, a diferencia de la interpretación de Gambier (1988:123), los escasos tiestos se interpretan aquí como intrusivos. Esa interpretación está basada en que el fechado es de un nivel con una profundidad de $190-200 \mathrm{~cm}$ en el cual se registra un solo tiesto y además se registraron un total de solo cinco tiestos entre 170$250 \mathrm{~cm}$ de profundidad (Gambier 1988: Cuadro 13). Por la misma razón, se excluyó el fechado GaK-4808 de Los Morillos, el cual proviene de un nivel con cinco tiestos, que son intrusivos según Gambier (1988:80). El modelo estima que la fase cerámica empezó en 2220-1920 cal. AP (68\% probabilidad, límite inicial) (Tabla 1, Figura 2). El amplio rango de error nos recuerda que el resultado es un rango de probabilidad que se podrá refinar.

\section{Mendoza}

En la provincia de Mendoza, los sitios con cerámica temprana se concentran en el norte. Los sitios del norte

\begin{tabular}{lcccccccc}
\hline \multicolumn{1}{c}{ Región } & Sitios & Fechados & $\begin{array}{c}\text { Eventos en la } \\
\text { fase cerámica }\end{array}$ & $\begin{array}{c}\text { Límite incial } \\
\text { Mediana }\end{array}$ & $68 \%$ & $95 \%$ & \pm & $\begin{array}{c}\text { Índice de acuerdo } \\
\text { al modelo }\end{array}$ \\
\hline San Juan & 5 & 11 & 10 & 2100 & $2220-1920$ & $2470-1840$ & 170 & 81 \\
Mendoza & 9 & 12 & 12 & 2140 & $2220-2040$ & $2370-1990$ & 100 & 104 \\
Chile semi árido & 8 & 15 & 9 & 1850 & $1900-1780$ & $1960-1740$ & 40 & 105 \\
Chile central & 15 & 31 & 29 & 2130 & $2190-2060$ & $2260-2010$ & 60 & 151 \\
Chile centro-sur & 10 & 11 & 11 & 2100 & $2150-2020$ & $2280-2000$ & 70 & 106 \\
Total & 47 & 80 & & & & & & \\
\hline
\end{tabular}

Tabla 1. Resumen de los resultados de los modelos bayesianos de cada áreas. El rango de error ( \pm ) solo sirve como aproximación general ya que los rangos de error calibrados no son simétricos.

Table 1. Summary of results from the Bayesian models for each area. The error range ( \pm ) is only useful as a general approximation, as calibrated error ranges are not symmetrical. 
Figura 2. Gráfico cronológico de las distribuciones de probabilidad de la fecha del primer uso de la cerámica en las cinco áreas (los límites iniciales de los modelos bayesianos). La parte sombreada de cada curva indica la distribución de probabilidad de $68 \%$. La línea vertical indica la mediana.

Figure 2. Timeline showing the distribution probabilities of the date of the earliest use of pottery in the five areas (starting boundaries from the Bayesian models). The shaded area within each curves indicates the $68 \%$ probability distribution. The vertical line indicates the median.

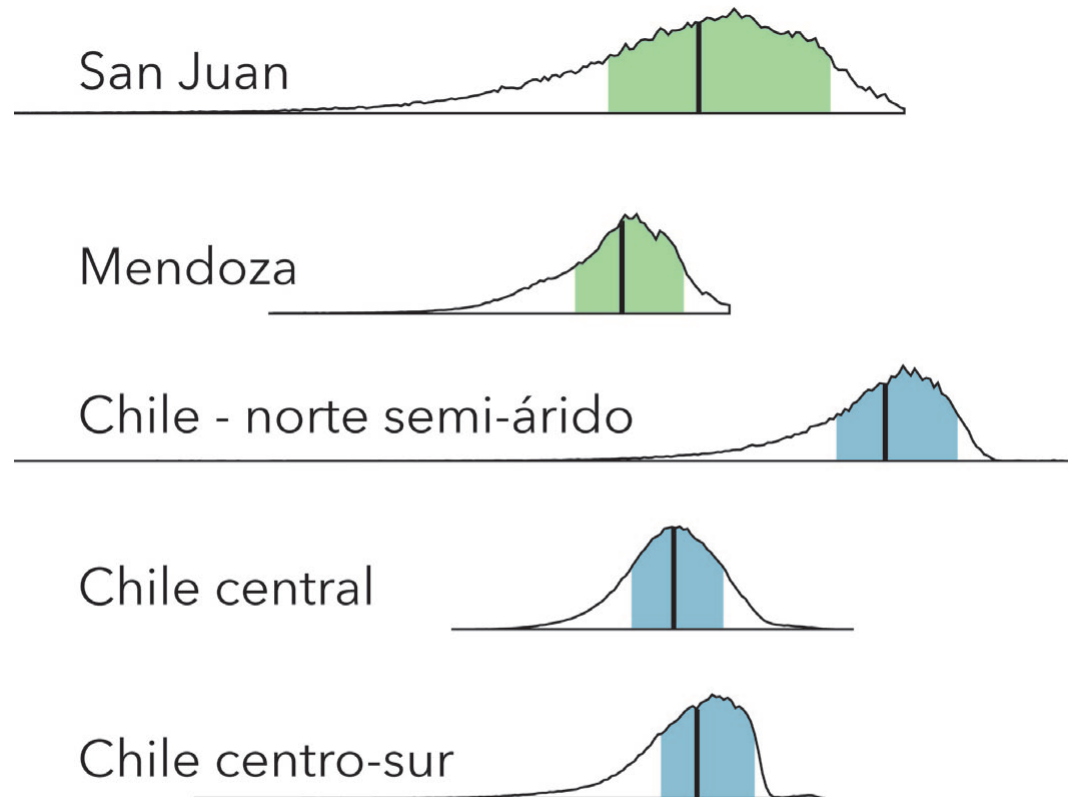

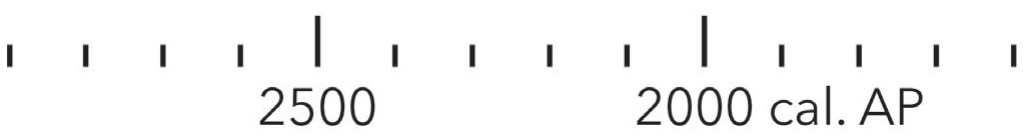

en zonas de altura son el Alero 1 del Arroyo Tambillos, Jagüel II y Rincón de los Helados (Bárcena 1998; Bárcena y Roig 1982; Chiavazza 1995). En el centro mendocino, el sitio de la Laguna del Diamante cuenta con escasos fragmentos, pero parece que existe un grado aceptable de asociación considerando el nivel bajo de impacto de procesos tafonómicos (Durán et al. 2006:93). Aquí se excluyeron los fechados del sitio Jagüel III porque el primer nivel con cerámica tiene diferentes dataciones que no tienen rangos de probabilidad similares (Bárcena 1998: 119-121, 126). En el sitio Plaza de Mendoza, se usó solo la muestra de carbón de una capa ocupacional como el fechado más confiable (Bárcena 1998:127-128). Existe un fechado muy similar, pero no publicado, de un contexto doméstico del sitio Memorial de la Bandera, también ubicado en la actual ciudad de Mendoza (Chaviazza 2011), que no forma parte del presente estudio. Otros entierros de la ciudad de Mendoza con edades similares (Gil et al. 2014:Tabla 2) tampoco fueron incluidos porque la asociación cerámica no es clara (Rusconi 1962, 1967). Los últimos tres fechados incorporados a la base analizada provienen de la zona de Barrancas en las cercanías del Río Mendoza. Uno proviene de un entierro y otros dos de un recinto semi-subterráneo en que se halló cerámica sobre el piso ocupacional (Gil et al. 2014: Tabla 2; Rusconi 1962:371; Marsh y Estrella 2015).

En el sur de la provincia se han registrado cinco sitios con cerámica temprana: Cañada de Cachi, La Olla, gruta de El Manzano, Cueva Palulo y Valle Hermoso 1 (Durán 2004:73-77,103-109; Giardina et al. 2015:56; Neme et al. 2011:256-258; Otaola y Llano 2015:224;
Sugrañes 2011:296, Tabla 1). En Cañada de Cachi, el nivel cerámico cuenta con un fechado contextual y uno por termoluminiscencia corregido por dosimetría (Dúran 2004:77). En el sitio Cueva Palulo, hay una capa entre 40 y $90 \mathrm{~cm}$ de profundidad que tiene tres fechados similares asociados a pocos fragmentos distribuidos de manera interrumpida (Otaola y Llano 2015: Figura 1, Tabla 1). El grado de asociación entre la cerámica y los fechados no es tan claro como en otros casos, pero aquí se sigue la interpretación de los excavadores del sitio de que los fechados se asocian con el uso de cerámica (G. Neme, comunicación personal 2016).

Los fechados radiocarbónicos del sur mendocino son muy similares a los del norte de la provincia, a pesar de la distancia entre sí. Suponiendo que los 16 fechados de Mendoza pertenecen a una fase de cerámica inicial, su límite inferior se estima en $2250-2100$ cal. AP (68\% probabilidad, límite inicial). Esta fecha se correlaciona con las sugerencias generales de trabajos anteriores que sintetizan datos de otros sitios y rasgos posiblemente asociados con el uso temprano de la alfarería (p. ej., Bárcena et al. 1985; García 1992:14-15; Durán y García 1989:54-56).

\section{Chile semi-árido}

En el norte semi-árido chileno, se trabajó con 15 fechados anteriores y posteriores de la cerámica inicial (Méndez et al. 2016; Troncoso et al. 2012, 2016; Rodríguez et al. 2000; Sanhueza et al. 2003; Vergara et al. 2012). Seis fechados radiocarbónicos provienen de niveles 
acerámicos tardíos de los sitios: Valle El Encanto, Alero Roca Fértil, Churque 4 y Techo Negro. Hay tres fechados radiocarbónicos de niveles cerámicos y seis de termoluminiscencia sobre tiestos cerámicos provenientes de los sitios Techo Negro, Valle El Encanto, Melina, Parcela Alejandro Mánquez, Los Mellizos, Bato 2 y Q. Batuco 2. Estas edades se agrupan en dos fases respectivas y el límite temporal entre ellas se estima por el modelo en 1900-1790 cal. AP (68\% probabilidad, límite inicial). Este modelo demuestra la ventaja de incorporar fechados anteriores al primer nivel con cerámica, que reducen el error del límite y llega a ser menor que los errores en otras áreas (Tabla 1).

Los datos obtenidos sugieren que el primer uso de la cerámica en el norte semi-árido fue varios siglos posterior al primer uso en regiones vecinas. Entre los pocos datos disponibles hay fechados de tres sitios con niveles acerámicos que se ubican en los mismos siglos en que la cerámica ya estaba claramente presente en Chile Central y San Juan. De esto surgen diferentes explicaciones: la primera es que pudo existir poco contacto entre las regiones estudiadas, por lo que los grupos del semi-árido habrían desconocido la cerámica. Sin embargo, es poco probable que grupos móviles separados por tan poca distancia no hayan tenido contacto ni al sur ni al este. La segunda posibilidad es que estos grupos sí conocían la cerámica pero decidieron no adoptarla por razones relacionadas a su entorno social y/o ambiente local. Esto genera un interrogante sobre las condiciones particulares del semi-árido chileno para estos siglos (Pavolvic 2003; Méndez et al. 2009; Troncoso y Pavlovic 2013), es decir, ¿qué factores llevaron a los grupos del semi-árido a no adoptar la cerámica al mismo tiempo que grupos en regiones vecinas? Este ejemplo nos recuerda que no son válidas las suposiciones que consideran que la cerámica se adopta de forma automática apenas está disponible y que sus ventajas son obvias.

\section{Chile Central}

La zona de Chile Central cuenta con el número más elevado de fechados tempranos (Falabella et al. 2015; Sanhueza et al. 2003; Sanhueza y Falabella 2000; Vasquéz et al. 1999). Existen 31 fechados de 15 sitios, cinco de los cuales se ubican en la costa (Curaumilla 1 y 2, Laguna El Peral-C, Arévalo y Arévalo 2). Otros nueve están concentrados en la tierras bajas cerca de la ciudad actual de Santiago: tres al norte (Radio Estación Naval, El Mercurio, y Parque La Quintrala) y seis al sur (Lonquén E80/4, VP5, CK2, CK5, CK9 y CK15). Hay un sitio de altura, Las Perdidas, muy cerca de Laguna del Diamante (LD-S2) del lado argentino, con una fecha similar (Cornejo y Sanhueza 2011: Tabla 1). En total para esta zona se han compilado 10 fechados radiocarbónicos y 21 fechados de termoluminiscencia relevantes. Los fechados sobre conchas marinas fueron calibrados con la curva de calibración Marine13 (Reimer et al. 2013), para poder hacer comparables los resultados. Los fechados más tempranos de todas las áreas consideradas provienen de los sitios costeros Curaumilla 1 y 2 en 2980-2760 cal. AP y 2690-2500 cal. AP. Estas dos fechas parecen pertenecer a un uso temprano y aislado de la tecnología, pero no queda clara su interpretación; aunque su asociación con recursos marinos seguiría un patrón visto en otros casos mundiales del uso inicial de la tecnología cerámica (Jordan y Zvelebil 2009a). El modelo bayesiano agrupa esos dos fechados en una fase anterior a la fase principal que contiene los demás fechados. El límite entre las dos fases estima la primera fecha de uso amplio de la cerámica en Chile Central en 2190-2060 cal. AP (68\% probabilidad, límite inicial).

La mayoría de los fechados en Chile Central son de termoluminiscencia y tienen rangos de error amplios de 140-200 años con un solo sigma, por lo que resulta clave recurrir a métodos bayesianos, sobre todo para estimar la fecha inicial de la fase. Sin usar estos métodos, la inspección visual simple o la suma de probabilidades tienden a suponer que las fases son más largas de lo que en realidad son (Bayliss et al. 2007:8-9; Bronk Ramsey 2003:89-91). Por ello se estimó el inicio de la fase de cerámica inicial demasiado temprano, en 300 AC (Falabella y Stehberg 1989). Algo similar ocurre con la fecha propuesta para el norte semi-árido, que también se estimó demasiado temprana, en 1 DC (Niemeyer et al. 1989). Problemas similares y adicionales surgen con el uso de las sumas de probabilidades de fechados, que son simples de elaborar y cada vez más comunes en la arqueología y también en los Andes del sur (p. ej., Barberena et al. 2016; Cornejo 2014; Falabella et al. 2015; Gil et al. 2015; Méndez et al. 2015). Sin embargo, no todas esas interpretaciones recientes hacen una consideración adecuada de las limitaciones del método (Bamforth y Grund 2012; Bayliss et al. 2007:9-11; Contreras y Meadows 2014; Williams 2012).

\section{Centro-sur de Chile}

En el centro-sur de Chile, entre los $36^{\circ}$ y $40^{\circ} \mathrm{S}$, se registraron 11 fechados asociados a cerámica temprana. Estos han sido compilados y discutidos recientemente por Campbell y Quiroz (2015) y Adán y Mera (2011). En trabajos anteriores, estos fechados fueron calificados como intrusivos por sus edades inesperadamente tempranas. Sin embargo las dataciones son coherentes entre sí y provienen de una variedad de sitios de la costa y cordillera estimados con los dos métodos diferentes (Adán y Mera 2011:14). El modelo incorpora cuatro fechados radiocarbónicos de los sitios habitacionales: Pichipehuenco-2, Flor de Lago-1 e Isla Mocha P25-1 (Adán y Mera 2011:14; Sánchez 1997) para esta región. Se incorporan además siete fechados de termoluminiscencia sobre tiestos cerámicos provienen de los sitios Isla Mocha, Talcahuano 1, Alero Quino 1 y Maicoyakuel (PU-220) (Bustos y Vergara 2001:75, 2004:285; Campbell y Quiroz 
2015:48-49; Dillehay 2014:213; Quiroz et al. 1997) y tres más publicados por Adán y Mera (2011: Tabla 2). Todos los sitios están ubicados en el norte de la región centro-sur, que podría representar un límite geográfico de la cerámica temprana en los $39.5^{\circ} \mathrm{S}$ aproximados, o simplemente un problema de muestreo por las escasas investigaciones en la región (Campbell y Quiroz 2015:49). Los 11 fechados se agrupan en una fase simple, como en el caso de Mendoza. El límite inicial se estima en 21502200 cal. AP (68\% probabilidad, límite inicial). El alto grado de acuerdo da como resultado un rango de error menor que en las otras regiones.

El centro-sur de Chile queda relativamente aislado de otros usos tempranos de la cerámica en los Andes del sur. Al este, en la provincia de Neuquén, el fechado más temprano asociado a la cerámica proviene de la Cueva Haichol (Fernández 1991) tienen una mediana calibrada de $1710 \mathrm{cal}$. AP (AC-077). Los otros fechados tempranos en Neuquén y el resto de Norpatagonia son unos siglos posteriores (p. ej. Sanguinetti de Bórmida et al. 2000; Fernández y Vitores 2007, 2008; Schuster 2014; Silveira et al. 2013). Lo mismo ocurre al norte del centro-sur, por ejemplo, en el valle de Maule, donde la cerámica más temprana se ha registrado en 750 años AP en adelante (Sanhueza et al. 1994). La falta de un registro similar en esas regiones vecinas representa un punto de partida para contrastar los procesos diferentes que operaban en distintas regiones.

\section{Discusión}

En cada una de las cinco áreas estudiadas se han usado modelos bayesianos para estimar la fecha de la cerámica inicial. El resultado principal de este trabajo es la coincidencia plena de las fechas en cuatro de las cinco áreas (excluyendo el norte-semi-árido chileno), que son estadísticamente indistinguibles, con medianas entre 2100 y 2180 cal. AP y un rango inclusivo de 2250-1920 cal. AP (68\% probabilidad, límites iniciales) (Tabla 1, Figura 2). Las distribuciones de probabilidad son amplias y bien solapadas y hacen imposible saber en cuál de esas cuatro regiones la cerámica se usó primero, exceptuando el uso aislado (en espacio y tiempo) en Curaumilla 1 y 2 en la Costa Pacífica. Con el grado actual de resolución cronológica, el primer uso de la cerámica en esas regiones fue simultáneo. Una correlación tan clara entre las fechas fue inesperada, sobre todo por las distancias entre los sitios. Se desprenden una serie de temas para discutir con este patrón espacio-temporal.

En primer lugar, el patrón no concuerda con una difusión tecnológica paulatina. Si hubo una difusión, fue sumamente rápida en un área enorme. Si existió un solo lugar en que se inventó primero y luego se difundió, pudo haber sido Chile Central, por tener más sitios y fechados tempranos que en las otras áreas. Sin embargo, el rango de probabilidad más temprana se encuentra en Mendoza.
Es factible que no hubo un solo lugar de origen, sino que fue inventada de forma independiente y paralela por grupos móviles como respuesta a un cambio mayor, por ejemplo, de clima o demografía. En este caso, los grupos de todas las áreas sabían de la cerámica antes, pero decidieron no adoptarla hasta que se enfrentaron con un cambio regional climático, de subsistencia o en el ámbito social.

Este escenario depende de las variaciones climáticas, por ejemplo en los 2000 o 2200 cal. AP, la intensidad de eventos ENSO en la región superó sus niveles actuales, según reconstrucciones concordantes del paleoclima con datos del océano Pacífico y sitios terrestres en Chile (Maldonado y Villagrán 2006:256; Rein et al. 2005:13; Riedinger et al. 2002). La ocurrencia de eventos ENSO más intensos debieron aumentar la inestabilidad de los recursos bióticos en una gran región, que pudo haber llevado a los grupos móviles a diversificar sus estrategias, utilizando la cerámica para poder manejar mejor los riesgos generados con recursos inestables.

Sin embargo, la explicación climática no responde la pregunta de por qué no se adoptó la cerámica en algunas áreas vecinas cerca de esa fecha, donde los impactos climáticos debieron ser similares. Sus habitantes hubiesen enfrentado siglos de eventos intensos de ENSO sin cerámica. Estos ejemplos recuerdan que un cambio regional en el clima no es suficiente para explicar por completo los cambios de las sociedades pasadas. Dentro de cada una de las comunidades afectadas por este cambio regional, la introducción de la cerámica pudo haber seguido caminos diferentes. Aclarar y discutir esas posibilidades fascinantes dependerá de los especialistas en cada región, quienes están trabajando para echar luz sobre los acontecimientos locales de una forma cada vez más profunda y robusta.

Al tratar los datos a una escala menor, resulta interesante introducir modelos sociales para entender por qué y cómo se adoptó la cerámica (Falabella y Planella 1989:4346, Figura 4; Sanhueza y Falabella 2000:42-44). Por ejemplo, Hayden (1995) propone que es adoptada por los miembros del grupo que buscan prestigio a través de la organización de eventos, con recipientes más decorados, con costos elevados de elaboración y comidas especiales que requieren preparación en recipientes especiales. Hay también varias propuestas economicistas (ver Brown 1989), las cuales sostienen que la cerámica había comenzado a ser utilizada para la preparación eficiente de semillas o para la extracción de grasa ósea de huesos, dado que requiere poca atención durante su larga cocción (Arnold 1985:128; Eerkens 2004:660). Entre esas y varias otras posibles explicaciones, habría que tener en cuenta tanto la ecología local en cuanto a los recursos y materias primas disponibles en las cercanías de cada sitio, como los procesos sociales donde se halla la cerámica temprana. 
Los resultados obtenidos sobre la fecha de la primera incorporación de la cerámica no son conclusiones finales que sirvan para marcar fases culturales o de desarrollo, sino que son datos que nos acercan a la inquietud antropológica sobre por qué estos grupos eligieron -o no- adoptar una nueva tecnología. Con esa pregunta en mente, resulta menos importante saber dógnde se inventó primero o si fue una tecnología inventada de forma independiente, prestada o difundida. Como una decisión de los grupos pasados que puede variar, queda abierta la posibilidad de que una vez incorporada la cerámica, esta pudo haber sido abandonada, al menos momentáneamente. Por ejemplo en Mendoza, luego de un uso inicial de la cerámica en varios sitios, hay unos siglos con menos señal de su uso que puede deberse a una falta de datos o quizás a cambios en la situación ecológica o social que llevó a no utilizar recipientes cerámicos. Los estudios detallados de la cerámica temprana pueden aclarar aspectos de grados variables de movilidad (Simms et al. 1997). Dicha perspectiva ayudará a elucidar las diferencias entre regiones en cuanto al uso de cerámica por grupos posteriores más sedentarios en el norte mientras que al sur la cerámica se mantenía como un elemento de grupos móviles hasta el contacto europeo.

\section{Conclusión}

Este trabajo discute la fecha inicial de la cerámica en cinco áreas de los Andes del sur. Los modelos bayesianos muestran que esta tecnología apareció en el registro arqueológico por primera vez en el mismo lapso cronológico a pesar de la enorme extensión del territorio analizado. En cuatro de las áreas analizadas, las fechas tienen rangos de error que son estadísticamente idénticos. A partir de un lapso breve alrededor de 2250$1920 \mathrm{cal}$. AP (68\% probabilidad, límites iniciales), los grupos humanos comenzaron a utilizar la cerámica, como en otros ejemplos de grupos móviles alrededor del mundo (Eerkens 2008). Es un patrón material y de coincidencia cronológica cuya explicación aún plantea un desafío. Refinar y aclarar la decisión de adoptar la cerámica por parte de los habitantes de los Andes del sur tiene implicaciones para entender uno de los cambios más profundos de la historia humana de la región. El próximo paso será evaluar las posibilidades que llevaron a grupos separados por tanta distancia a decidir adoptar la misma tecnología al mismo tiempo. La investigación futura tendrá en cuenta la importancia de esos siglos al momento de excavar, establecer relaciones claras entre las muestras fechadas, evaluar la ecología local de sitios con cerámica temprana, testear modelos sociales para su adopción y comparar datos similares de áreas vecinas. De esa forma se podrá 'dar bordadas' (sensu Wylie 1989:10) entre diferentes escalas, datos y modelos hacia un panorama del pasado más robusto.

\section{Agradecimientos}

Una versión anterior de este trabajo fue presentada en las VI Jornadas Arqueológicas Cuyanas y se agradece al comité organizador de San Rafael y Los Reyunos, a los organizadores del simposio "Actualización de los estudios cerámicos en el centro oeste argentino" y a los editores de la revista por la invitación a participar en este número especial. No hubiese sido posible sin el interés y la ayuda de varios colegas argentinos y chilenos que me ayudaron a encontrar citas y datos de muchos sitios, entre ellos Roberto Campbell, Horacio Chiavazza, Luis Cornejo, Víctor Durán, Andrés Izeta, César Méndez y Andrés Troncoso. Agradezco a Ramiro Barberena, Valeria Cortegoso, Adolfo Gil, Stella Moreiras, Gustavo Neme y dos evaluadores anónimos por sus comentarios que mejoraron bastante el manuscrito. El estudio fue realizado con el apoyo de CONICET y el querido equipo del Laboratorio de PaleoEcología Humana.

\section{Bibliografía}

Adán, L. \& R. Moreno. 2011. Variabilidad interna en el Alfarero Temprano del centro-sur de Chile: El complejo Pitrén en el valle central del Cautín y el sector lacustre andino. Chungara 43:3-23.

Arnold, D. 1985. Ceramic theory and cultural process. Cambridge: Cambridge University Press.

Babot, M. 2006. El papel de la molienda en la transición hacia la producción agropastoril: Un análisis desde la puna meridional argentina. Estudios Atacameños 32:75-92.

Babot, M. 2011. Cazadores-recolectores de los andes centrosur y procesamiento vegetal: Una discusión desde la puna meridional argentina (ca. 7.000-3.200 años AP). Chungara 43:413-432.

Bamforth, D.B. \& B. Grund. 2012. Radiocarbon calibration curves, summed probability distributions, and early Paleoindian population trends in North America. Journal of Archaeological Science 39:1768-1774.

Bandy, M.S. 2001. Population and history in the ancient Titicaca Basin. Tesis doctoral inédita. University of California, Berkeley: Department of Anthropology.

Barberena, R., C. Méndez \& M.E. de Porras. 2016. Zooming out archaeological discontinuities: The meaning of mid-Holocene troughs in South American Deserts. Journal of Anthropological Archaeology. En prensa.

Bárcena, J.R. 1998. Arqueología de Mendoza. Las dataciones absolutas y sus alcances. EDIUNC, Mendoza.

Bárcena, J.R. \& F. Roig. 1982. Investigaciones arqueológicas en el área puneña de Mendoza, con especial referencia a Tephrocactus andicola (Cactaceae) como nuevo recurso alimentario. Relaciones de la Sociedad Argentina de Antropología 14:85-107.

Bárcena, J.R., F. Roig \& V. Roig. 1985. Aportes arqueofitozoológicos para la prehistoria del NO de la provincia de Mendoza: La excavación de Agua de la Tinaja I. Trabajos de Prehistoria 42:311-362. 
Barnett, W. \& J. Hoopes. 1995. The emergence of pottery: technology and innovation in ancient societies.

Bayliss, A. 2009. Rolling out revolution: Using radiocarbon dating in archaeology. Radiocarbon 51:123-147.

Bayliss, A., C. Bronk Ramsey, J. van der Plicht \& A. Whittle. 2007. Bradshaw and Bayes: Towards a timetable for the Neolithic. Cambridge Archaeological Journal 17:1-28.

Bronk Ramsey, C. 2003. Punctuated dynamic equilibria: A model for chronological analysis. En: R. Bentley, H. Maschner (editores), Complex systems and archaeology: Empirical and theoretical applications. Salt Lake City, USA: University of Utah Press. p. 85-92.

Bronk Ramsey, C. 2008. Radiocarbon dating: revolutions in understanding. Archaeometry 50 (2): 249-275.

Bronk Ramsey, C.B. 2009. Bayesian analysis of radiocarbon dates. Radiocarbon 51:337-360.

Browman, D. 1981. New light on Andean Tiwanaku. American Scientist 69:408-419.

Buck, C., W. Cavanagh \& C. Litton. 1996. Bayesian approach to interpreting archaeological data. Chichester, UK: John Wiley \& Sons.

Brown, J.A. 1989. The beginnings of pottery as an economic process. En: S. van der Leuw, R. Torrence (editores), What's new? A closer look at the process of innovation. London: Unwin Hyman. p. 203-224.

Buck, C. \& A. Millard. 2004. Tools for Constructing Chronologies: Crossing Disciplinary Boundaries. London: Springer.

Buck, C., W. Cavanagh \& C. Litton. 1996. Bayesian approach to interpreting archaeological data. Chichester, UK: John Wiley \& Sons.

Bustos, V. \& N. Vergara. 2001. Evolución de las prácticas mortuorias en el litoral de la octava región. Chungara 33:73-78.

Bustos, V. \& N. Vergara. 2004. Modelos de ocupación temprana en la Bahía de Concepción y Golfo de Arauco. Chungara 36:283-288.

Campbell, R. \& D. Quiroz. 2015. Chronological database for southern Chile $\left(35^{\circ} 30^{\prime}-42^{\circ} \mathrm{S}\right), \sim 33,000$ BP to present: human implications and archaeological biases. Quaternary International 356:39-53.

Chiavazza, H. 1995. Estudios arqueológicos en el sitio 'Rincón de los Helados. Ocupación multicomponente en el noreste de Pampa de Canota, Mendoza. Tesis de licenciatura inédita. Universidad Nacional de Cuyo, Mendoza.

Chiavazza, H. 2011. Restos de una pequeña aldea milenaria. La Ciudad: Revista de la Municipalidad de la Ciudad de Mendoza 3:4-5.

Childe, G. 1925. The dawn of European civilization. London: Kegan Paul.
Contreras, D. \& J. Meadows. 2014. Summed radiocarbon calibrations as a population proxy: A critical evaluation using a realistic simulation approach. Journal of Archaeological Science 52:591-608.

Cornejo, L. 2014. Sobre la cronología de la imposición cuzqueña en Chile. Estudios Atacameños 47:101-116.

Cornejo, L. \& L. Sanhueza. 2011. North and south: Huntergatherer communities in the Andes mountains in central Chile. Latin American Antiquity 22:487-504.

Dillehay, T. D. 2014. The teleoscopic polity: Andean patriarchy and materiality. Contributions to Global Historical Archaeology. Cham, Switzerland: Springer.

Durán, V. 2004. Poblaciones indígenas de Malargüe: Su arqueología e historia. Mendoza: Universidad Nacional de Cuyo.

Durán,V. \& C. García. 1989. Ocupaciones agroalfareras en el sitio Agua de la Cueva sector norte (NO de Mendoza). Estudios Regionales 3:29-64.

Durán, V., G. Neme, V. Cortegoso \& A. Gil. 2006. Arqueología del área natural protegida Laguna del Diamante (Mendoza, Argentina). En: V. Durán, Cortegoso V. (editores), Arqueología y Ambiente de Áreas Naturales Protegidas de la Provincia de Mendoza. Mendoza: EDIUNC. p. 81-134.

Eerkens, J. W. 2003. Residential mobility and pottery use in the western great basin. Current Anthropology 44:728-738.

Eerkens, J. W. 2004. Privatization, Small-Seed Intensification, and the Origins of Pottery in the Western Great Basin. American Antiquity, 69(4), 653-670

Eerkens, J. W. 2008. Nomadic potters: Relationships between ceramic technologies and mobility strategies. En: H. Barnard, W. Wendrich (editores), The archaeology of mobility: Old world and new world nomadism, Los Angeles: Cotsen Institute of Archaeology. p. 307-326.

Falabella, G. \& M. Planella. 1989. Alfarería temprana en chile central: Un modelo de interpretación. Paleoetnología 5:41-60.

Falabella, F. \& R. Stehberg. 1989. Los inicios del desarrollo agrícola y alfarero: zona central (300 a. C. A 900 d. C.). En Hidalgo J., Schiappacasse V., Niemeyer H., Aldunate C. y Solimano I. (editores), Culturas de chile: Prehistoria, desde sus orígenes hasta los albores de la conquista. Santiago: Andrés Bello. p. 227-263.

Falabella, F., L.E. Cornejo, L. Sanhueza \& I. Correa. 2015. Trends in thermoluminescence date distributions for the Angostura micro region in central Chile. Quaternary International 356:2738.

Fernández, J. 1989. Ocupaciones alfareras (2,860 \pm 160 años AP) en la Cueva de Cristóbal, Puna de Jujuy, Argentina. Relaciones de la Sociedad Argentina de Antropología 17:139-178.

Fernández, J. 1991. La cueva de Haichol. Arqueología de los pinares cordilleranos del Neuquén. Anales de Arqueología y Etnología 43-45:1-740. 
Fernández, M. \& M. Vitores. 2007. Tecnología cerámica de la cuenca inferior del arroyo Pichileufú, provincia de Río Negro. Ponencia presentada en el XVI Congreso Nacional de Arqueología Argentina, San Salvador de Jujuy.

Fernández, M. \& M. Vitores. 2008. Distribución de la cerámica arqueológica en la cuenca superior y media del Río Limay. Ponencia presentada en las Terceras Jornadas de Historia de la Patagonia, San Carlos de Bariloche.

Gambier, M. 1977. La cultura de Ansilta. Instituto de Investigaciones Arqueológicas y Museo, San Juan.

Gambier, M. 1988. La fase cultural Punta del Barro. Instituto de Investigaciones Arqueológicas y Museo, San Juan

García, A. 1992. Hacia un ordenamiento preliminar de las ocupaciones prehistóricas agrícolas precerámicas y agroalfareras en el NO de Mendoza. Estudios Regionales 10:7-34.

García, L. 1995. Las primeras cerámicas en la puna de Jujuy. Cuadernos de la Facultad de Humanidades Y Ciencias Sociales, Universidad Nacional de Jujuy 5:75-81.

García, L.C. \& P.S. Higa. 2014. Un caso de estudio sobre sociedades productoras de alimentos iniciales a plenas en la Provincia de Jujuy. En: G. Cassiodoro, A. Re, D. Rindel (editores), Integración de diferentes líneas de evidencia en la arqueología argentina. Buenos Aires: Aspha. p. 17-39.

Giardina, M., M. Corbat, E. Peralta, G. Cochero, F. Franchetti, L. Salgán \& A. Gil. 2015. El registro arqueológico en el sitio La Olla (San Rafael, Mendoza): Implicaciones para las ocupaciones humanas en el valle medio del río Atuel. Revista del Museo de Antropología 8:51-66.

Gil, A., R. Villalba, A. Ugan, V. Cortegoso, G. Neme, C.T. Michieli, P. Novellino \& V. Durán. 2014. Isotopic evidence on human bone for declining maize consumption during the Little Ice Age in central western Argentina. Journal of Archaeological Science 49:213-227.

Gil, A., M. Giardina, G. Neme \& A. Ugan. 2015. Demografía humana e incorporación de cultígenos en el centro occidente argentino: Explorando tendencias en las fechas radiocarbónicas. Revista Española de Antropología Americana 44:523-533.

Görsdorf, J. \& M. Reindel. 2002. Radiocarbon dating of the Nasca settlements Los Molinos and La Muña in Palpa, Peru. Geochronometria 21:151-156.

Greco, C. 2012. Integración de los datos arqueológicos, radiocarbónicos y geofísicos para la construcción de una cronología de Yocavil y alrededores. Tesis doctoral inédita. Facultad de Filosofía y Letras: Universidad de Buenos Aires.

Hastorf, C. A. 2008. The Formative Period in the Titicaca Basin. En: H. Silverman, W. Isbell (editores), The handbook of South American Archaeology. Springer, New York. p. 545-561.

Hayden, B. 1995. The emergence of prestige technologies and pottery. En: W. Barnett, J. Hoopes (editors), The emergence of pottery: Technology and innovation in ancient societies. Washington, DC: Smithsonian Institution. p. 257-266.
Hocsman, S. 2002. ¿Cazadores-recolectores complejos en la puna meridional Argentina? Entrelazando evidencias del registro arqueológico de la microrregión de Antofagasta de la Sierra (Catamarca). Relaciones de la Sociedad Argentina de Antropología 27:193-214.

Hocsman, S., M. Babot, M. Cremonte, M. Aguirre, A. Gerónimo \& A. Calisaya. 2012. Sobre límites difusos: Hacia el "Formativo" en la puna argentina y su borde oriental. Presentado en el Encuentro Arqueología del Período Formativo en Argentina, Tafí del Valle.

Hogg, A., Q. Hua, P. Blackwell, M. Niu, C. Buck, T. Guilderson, T. Heaton, J. Palmer, P. Reimer \& R. Reimer. 2013. SHCal13 southern hemisphere calibration, 0-50,000 cal yr BP. Radiocarbon 55:1889-1903.

Jordan, P. \& M. Zvelebil. 2009a. Ex oriente lux: The prehistory of hunter-gatherer ceramic dispersals. En: P. Jordan, M. Zvelebil (editores), Ceramics before farming: The dispersal of pottery among prehistoric Eurasian hunter-gatherers. Walnut Creek, CA: Left Coast Press. p. 33-90.

Jordan, P. \& M. Zvelebil. 2009b (editores). Ceramics before farming: The dispersal of pottery among prehistoric Eurasian hunter-gatherers. Walnut Creek, CA: Left Coast Press.

Kaner, S. 2009. Long-term innovation: The appearance and spread of pottery in the Japanese archipelago. En: P. Jordan, M. Zvelebil (editores), Ceramics before farming: The dispersal of pottery among prehistoric Eurasian hunter-gatherers. Walnut Creek, CA: Left Coast Press. p. 91-120.

Koons, M. \& B. Alex. 2014. Revised Moche chronology based on Bayesian models of reliable radiocarbon dates. Radiocarbon 56:1039-1055.

Korpisaari, A., M. Oinonen \& J. Chacama. 2014. A reevaluation of the absolute chronology of Cabuza and related ceramic styles of the Azapa valley, northern Chile. Latin American Antiquity 25:409-426.

Korstanje, M., M. Lazzari, M. Basile, F. Bugliani, V. Lema, L. Pereyra Domingorena \& M. Quesada. 2015. Crónicas materiales precolombinas. Arqueología de los primeros poblados del noroeste argentino. Buenos Aires: Sociedad Argentina de Antropología.

Lavallée D. 2006. Secuencias y consecuencias de algunos procesos de neolitización en los Andes. Estudios Atacameños 32:35-41.

Lema, V. 2010. Procesos de domesticación vegetal en el pasado prehispánico del noroeste argentino: Estudio de las prácticas más allá de los orígenes. Relaciones de la Sociedad Argentina de Antropología 35:121-142.

Lema, V. 2014. Boceto para un esquema: Domesticación y agricultura temprana en el noroeste argentino. Revista Española de Antropología Americana 44:465-494.

López Campeny, S.M.L. \& A.S. Romano. 2012. Remodelando el Formativo. Aportes para una discusión de los procesos locales en las comunidades agropastoriles tempranas de Antofagasta de la Sierra (Catamarca, Argentina). Encuentro Arqueología del 
Período Formativo en Argentina, Tafí del Valle.

Lumbreras, L.G. 2006. Un Formativo sin cerámica y cerámica preformativa. Estudios Atacameños 32:11-34.

Maldonado, A. \& C. Villagrán. 2006. Climate variability over the last $9900 \mathrm{cal}$ yr BP from a swamp forest pollen record along the semiarid coast of Chile. Quaternary Research 66:246-258.

Marsh, E. 2012. A Bayesian re-assessment of the earliest radiocarbon dates from Tiwanaku, Bolivia. Radiocarbon 54:203-218.

Marsh, E. 2015. The emergence of agropastoralism: Accelerated ecocultural change on the Andean altiplano, 3540-3120 cal BP. Environmental Archaeology 20:13-29.

Marsh, E. 2016. The disappearing desert and the emergence of agropastoralism: An adaptive cycle of rapid change in the mid-Holocene Lake Titicaca Basin (Peru-Bolivia). Quaternary International 422:123-134.

Marsh, E. \& V. Cortegoso. 2014. Refinando la cronología del valle de Potrerillos mediante modelos de Bayes. En: V. Cortegoso, V. Durán \& A. Gasco (compiladores), Arqueología de Ambientes de Altura de Mendoza y San Juan (Argentina). Mendoza: EDIUNC. p. 57-79.

Marsh, E., D. Estrella. 2015. Una casa-pozo temprana en Barrancas, Maipú. Ponencia presentada en las II Jornadas Salvador Canals Frau, Universidad Nacional de Uuyo, Mendoza.

Marshall, Y. 2006. Introduction: Adopting a sedentary lifeway. World Archaeology 38:153-163.

Méndez, C., M. Troncoso, D. Jackson \& D. Pavlovic. 2009. Movilidad y uso del espacio entre cazadores-recolectores tardíos en espacios cordilleranos del norte semiárido de chile. Intersecciones en Antropología 10:311-326.

Méndez, C., A. Gil, G. Neme, A. Nuevo Delaunay, V. Cortegoso, C. Huidobro, V. Durán \& A. Maldonado. 2015. Mid-Holocene radiocarbon ages in the subtropical Andes $\left(29^{\circ}-35^{\circ} \mathrm{S}\right)$, climatic change and implications for human space organization. Quaternary International 356:15-26.

Méndez, C., S. Grasset, D. Jackson, A. Troncoso \& B. Santander. 2016. Ocupaciones humanas del Holoceno Medio en los Andes del norte semiárido de Chile ( $31^{\circ} \mathrm{S}$, Combarbalá): Función del sitio e implicancias para el uso regional del espacio. Chungara 48:225-241.

Michczyński, A.\& A. Pazdur. 2003. The method of combining radiocarbon dates and other information in application to study the chronologies of archaeological sites. Geochronometria 22:41-46.

Michczyński, A., P. Eeckhout \& A. Pazdur. 2003. ${ }^{14} \mathrm{C}$ absolute chronology of pyramid III and the dynastic model at Pachacamac, Peru. Radiocarbon 45:59-73.

Michczyński, A., P. Eeckhout, A. Pazdur \& J. Pawlyta. 2007. Radiocarbon dating of the Temple of the Monkey-the next step towards a comprehensive absolute chronology of Pachacamac, Peru. Radiocarbon 49:565-578.
Neme, G., A. Gil, R. Garvey, C. Llano, A. Zangrando, F. Franchetti, C. de Francesco \& C.T. Michieli. 2011. El registro arqueológico de la gruta de El Manzano y sus implicancias para la arqueología de Nordpatagonia. Magallania 39:243-265.

Niemeyer, H., G. Castillo \& M. Cervellino. 1989. Los primeros ceramistas del norte chico: Complejo El Molle (0 a 800 d.C.). En J. Hidalgo, V. Schiappacasse, H. Niemeyer, C. Aldunate y I. Solimano (editores), Culturas de chile: Prehistoria, desde sus orígenes hasta los albores de la conquista. Santiago: Andrés Bello. p. 227-263.

Núñez, L. \& C. Santoro. 2011. El tránsito Arcaico-Formativo en la circumpuna y valles occidentales del centro sur andino: Hacia los cambios "neolíticos". Chungara 43:487-530.

Núñez, L., V. McRostie \& I. Cartajena. 2009. Consideraciones sobre la recolección vegetal y la horticultura durante el Formativo temprano en el sureste de la cuenca de atacama. Darwiniana 47:56-75.

Ogburn, D. 2012. Reconceiving the chronology of Inca imperial expansion. Radiocarbon 54:219-237.

Olivera, D. 2001. Sociedades agropastoriles tempranas: El Formativo inferior del noroeste argentino. En: E. Berberián, A. Nielsen (editores), Historia argentina prehispánica. Córdoba: Brujas. p. 83-125.

Olivera, D., P. Escola, A. Elías, S. Pérez, P. Tchilinguirian, P. Salminci, M. Pérez, L. Grana, J.G. Lett-Brown, V. Aixa, V.K. Galván \& P. Miranda. 2012. El Formativo en la puna meridional: De la opción productiva a las sociedades agropastoriles plenas. Encuentro Arqueología del Período Formativo en Argentina, Tafí del Valle.

Olivera, D., A. Elías, M. Pérez \& P. Salminci. 2015. Corral Grande 1 y Arroyo Seco: Nuevos aportes al Formativo de Antofagasta de la Sierra (Provincia de Catamarca, puna meridional Argentina). Comechingonia 19:37-66.

Otaola, C. \& C. Llano. 2015. Consumo de vegetales y animales en el sur de Mendoza: El caso del sitio Cueva Palulo. Intersecciones en Antropología 16:221-235.

Pavlovic, D. 2003. Dejando atrás la tierra de nadie: asentamientos, contextos y movilidad de las comunidades alfareras tempranas del Choapa. Werken 5:39-46.

Pluciennik, M. 2002. The invention of hunter-gatherers in seventeenth-century Europe. Archaeological Dialogues 9:98151.

Pluciennik, M. 2005. Social Evolution. London: Duckworth.

Politis, G., G. Martínez \& M. Bonomo. 2001. Alfarería temprana en sitios de cazadores-recolectores de la región pampeana (Argentina). Latin American Antiquity 12:167-181.

Quiroz, D., M. Vásquez \& M. Sánchez. 1997. Quino-1, un sitio alfarero temprano en la región centro-sur: noticias y comentarios para un fechado. Boletín de la Sociedad Chilena de Arqueología 24:49-52

Reimer, P.J., E. Bard, A. Bayliss, J.W. Beck, P.G. Blackwell, C.B. 
Ramsey, P.M. Grootes, T.P. Guilderson, H. Haflidason, I. Hajdas, C. Hatté, T.J. Heaton, D.L. Hoffma, A.G. Hogg, K.A. Hughen, K.F. Kaiser, B. Kromer, S. Manning, M. Niu, R.W. Reimer, D.A. Richards, E.M. Scott, J.R. Southon, R.A. Staff, C.S.M. Turney \& J. van der Plicht. 2013. IntCal13 and Marine13 radiocarbon age calibration curves $0-50,000$ years cal BP. Radiocarbon 55:1869-1887.

Rein, B., A. Lückge, L. Reinhardt, F. Sirocko, A. Wolf \& W. Dullo. 2005. El Niño variability off Peru during the last 20,000 years. Paleoceanography 20:PA4003.

Rice, P.M. 1999. On the origins of pottery. Journal of Archaeological Method and Theory 6: 1-54.

Riedinger, M.A., M. Steinitz-Kannan, W.M. Last \& M. Brenner. 2002. A $\sim 6100{ }^{14} \mathrm{C}$ yr record of El Niño activity from the Galápagos islands. Journal of Paleolimnology 27:1-7.

Rodríguez, J., A. Troncoso, C. Becker, P. González \& D. Pavlovic. 2000. Ocupaciones prehispanas en la cuenca del río Illapel. En: Actas del XIV Congreso Nacional de Arqueología Chilena. Copiapó, Chile: Museo Regional de Atacama. p. 331-342.

Rudebeck, E. 2000. Tilling nature, harvesting culture. Exploring images of the human being in the transition to agriculture. Stockholm: Almqvist and Wiksell.

Rusconi, C. 1962. Arqueología. Poblaciones pre y posthispanicas de Mendoza, Vol. III. Mendoza.

Rusconi, C. 1967. Restos indígenas hallados en una excavación de Las Heras (Mendoza). Revista del Museo de Historia Natural de Mendoza "Juan C. Moyano" 19:3-13.

Sánchez, M. 1997. El período alfarero en la Isla Mocha. En: D. Quiroz, M. Sánchez (compiladores), La Isla de las Palabras Rotas. Santiago: Centro de Investigaciones Diego Barros Arana. p. 103-131.

Sanguinetti de Bórmida, A., A. Chauvin, D. Curzio, E.A. Crivelli Montero \& M. Lezcano. 2000. Arqueología de rescate en el alero La Marcelina 1, Pcia. de Río Negro. Actas de III Congreso Argentino de Americanistas (1999). Vol III. Buenos Aires. p. 351-372.

Sanhueza, L., F. Vilches, C. Rees, C. Westfall \& A. Seelenfreund. 1994. Ocupaciones arqueológicas de la precordillera y cordillera de la cuenca del río Maule: Un panorama general. En: Actas del $2^{\circ}$ taller de arqueología de chile central. p. 1-17.

Sanhueza, L. \& F. Falabella. 2000. Las comunidades alfareras iniciales en Chile central. Revista Chilena de Antropología 15:29-47.

Sanhueza, L., M. Vásquez \& F. Falabella. 2003. Las sociedades alfareras tempranas de la cuenca de Santiago. Chungara 35:23-50.

Schuster, V. 2014. La organización tecnológica de la cerámica de cazadores-recolectores. Relaciones de la Sociedad Argentina de Antropología 39:203-231.

Silveira, M.J., L.G. López \& V. Aldazabal. 2013. El uso del espacio durante el Holoceno Tardío - últimos 3500 años - en el bosque andino de Patagonia septentrional, Lago Traful, sudoeste de la provincia de Neuquén. Anuario de Arqueología 5:85-101.

Simms, S., J. Bright \& A. Ugan. 1997. Plain-ware ceramics and residential mobility: a case study from the Great Basin. Journal of Archaeological Science 24:779-792.

Smith, B.D. 2001. Low-level food production. Journal of Archaeological Research 9:1-43.

Smith, A.B. 2005. African herders: emergence of pastoral traditions. Walnut Creek, CA: AltaMira Press.

Sugrañes, N. 2011. Tecnología cerámica y estrategias de movilidad entre cazadores-recolectores de altura: El caso del sitio Valle Hermoso 1 (Malargüe, Mendoza). Intersecciones en Antropología 12:293-304.

Troncoso, A., P. Larach, S. Alfaro, D. Pascual \& D. Pavolvic. 2012. Nuevos antecedentes para el período Alfarero Temprano en el valle del Choapa: El sitio Los Mellizos (cuenca superior del Río Illapel). En: Actas del XVIII congreso nacional de arqueología chilena. Santiago: Sociedad Chilena de Arqueología. p. 309-319.

Troncoso, A. \& D. Pavlovic. 2013. Historia, saberes y prácticas: Un ensayo sobre el desarrollo de las comunidades alfareras del norte semiárido chileno. Chungara 27:101-140.

Troncoso, A., F.Vergara, D. Pavlovic, P. González, M. Pino, P. Larach, A. Escudero, N. La Mura, F. Moya, I. Pérez, R. Gutiérrez, D. Pascual, C. Belmar, M. Basile, P. López, C. Dávila, M.J.Vásquez \& P. Urzúa. 2016. Dinámica espacial y temporal de las ocupaciones prehispánicas en la cuenca hidrográfica del río Limarí (30 lat. S.). Chungara 48:199-224.

Unkel, U., B. Kromer, M. Reindel, L. Wacker \& G. Wagner. 2007. A chronology of the pre-columbian Paracas and Nacsa cultures in south Peru based on AMS ${ }^{14} \mathrm{C}$ dating. Radiocarbon 49:551-564.

Vásquez, M., L. Sanhueza \& F. Falabella. 1999. Nuevos fechados para el período Agroalfarero Temprano en la cuenca de Santiago: presentación y discusión. Boletín de la Sociedad Chilena de Arqueología 28:9-18.

Vergara, F., L. Vargas, J. Hernández, M. Rebolledo, F. Fernández \& P. Peralta. 2012. Grupos cazadores recolectores con cerámica en el norte semiárido de Chile. Ponencia presentada en el XIX Congreso Nacional de Arqueología Chilena, Arica.

Williams, A. 2012. The use of summed radiocarbon probability distributions in archaeology: a review of methods. Journal of Archaeological Science 39:578-589.

Wood, R. 2015. From revolution to convention: The past, present and future of radiocarbon dating. Journal of Archaeological Science 56:61-72.

Wylie, A. 1989. Archaeological cables and tacking: The implications of practice for Bernstein's 'options beyond objectivism and relativism'. Philosophy of the Social Sciences 251:1-18.

Yacobaccio, H. \& A. Korstanje. 2007. Los procesos de domesticación vegetal y animal: un aporte a la discusión argentina en los últimos 70 años. Relaciones de la Sociedad Argentina de Antropología 32:191-216. 